\title{
A Preliminary Study for Exploring High-speed Train Driver Fatigue Using Eye-gaze Cue
}

\author{
Ronghui Yan, Cheng Wu*and Yiming Wang \\ School of Urban Rail Transportation, Soochow University, Suzhou, China \\ ${ }^{*}$ Corresponding author
}

\begin{abstract}
High-speed railway is seeking for a higher safety level than other public transports because of more passengers and higher speed. However, sleepiness and fatigue occur more frequently for train drivers due to longer operation time and irregular shift schedule. In the paper, we focus on study the particularity of train drivers' fatigue under the environment of high-speed railway. We observed operation performance and evaluated the degree of fatigue using train drivers' visual characteristics, while they conducted various tasks on high-speed railway driver simulator. By analyzing self-report sleepiness scales, detection distance and eye movement data, we found that pupil diameter, blink time and fixation percentage of fatigue drivers were significantly different from that of sober drivers. Meanwhile, we revealed obvious individual difference of train drivers and proposed a personalized database for train drivers' fatigue identification. These quantitative results can be used as a preliminary study for designing human-train interface of highspeed railway.
\end{abstract}

Keywords-eye tracking; fatigue; cognitive performance; individual difference; statistical analysis

\section{INTRODUCTION}

In recent years, high-speed railway is rapidly growing in the world. When people enjoy the benefits from high-speed railway, such as time-saving, convenience and comfort, public attention on rail safety is also greatly raised. Train drivers play a key role on the rail operation and their physiological and psychological states are important factors to ensure driving safety.

Sleepiness and fatigue at work occur frequently in rail transportation, with half of the train drivers and traffic controllers severely fatigue during night shift [1]. Investigations conducted by Australian Transport Safety Bureau (ATSB) revealed that work-related fatigue was a major factor leading to the train accidents or incidents in Tarcoola [2], Sydney [3], Mt Druitt [4].

The impact of operator fatigue on safety performance is a major issue in the railroad industry [5]. The obvious negative impact is due to the particularity of train drivers' work compared with other professional drivers. High psychological and cognitive requirements, high workload and work hours, and irregular shift schedules make it easier for train drivers to fall asleep during work time [6, 7, 8]. Different dynamic forces, upcoming terrain, traffic signs and communication need drivers' constant surveillance [1]. Automated vehicle control and monitoring systems reduce human operator activity, also cause monotony and result in loss of driver vigilance $[9,10]$.
In order to monitor train drivers' fatigue state, the biobehavioral characteristics of train drivers can be used as an indicator of fatigue. Train driving is primarily a visual task. Drivers in fatigue state exhibit certain eye behavior, which can be reflected by eyelid movement, gaze and pupil size. Eye features are widely used in vehicle fatigue monitoring systems. Good results have been reported with techniques that monitor and combine eyelid movement, gaze movement, head movement, and facial expression systematically using a probabilistic model to predict driver fatigue [11]. However, the prevalence and the effect of fatigue on the train drivers' eye behaviors are not clear. Moreover, few studies in driver fatigue detection area took inter-individual differences into consideration Therefore, with the aim of studying the eye behaviors characteristics of train drivers caused by fatigue, we conducted statistical analysis on pupil size, eye blinks and fixation. We analyzed train drivers' individual difference of eye movement characteristics and proposed personalized database hypothesis for train drivers' fatigue identification.

This paper is organized as follows. Section 2 describes the proposed method, comprising participants, apparatus, stimuli, procedure, and data processing. Section 3 presents experiment validation and feature extraction. Section 4 presents statistical analysis results. Finally, Section 5 provides conclusions.

\section{METHOD}

\section{A. Participants}

With regard to individual representativeness, all participants were with traffic engineering disciplines and had to pass the required credits of theory and practice parts in rail operation courses.

A total of 9 participants applied for the experiment. As a result of the above criteria, there were 6 participants qualified, including 3 women and 3 men, with an age range of 20-25 years $(M=21, S D=1.4)$. All participants had normal color vision and hearing, and had no sleep-related disease. They gave consent to the experiment and received a reward for completing the experiment.

\section{B. Apparatus and Stimuli}

The study was conducted in a bullet train driving simulator. We designed railway environment and safety critical events by setting simulated herds on the track. The simulation video was projected onto the screen $283 \mathrm{cmaway}$ from the participant's eyes at a resolution of $768 \times 1024$.Eye movement data were collected by Tobii X2-60 remote eye tracker. The eye tracker was mounted on the dashboard in front of the driver at a distance of $65 \mathrm{~cm}$ without disturbing his visual field. The 
illumination and temperature were controlled constant and comfortable for all participants.

Sleepiness was assessed using the Karolinska Sleepiness Scale (KSS) and sleep diaries. KSS is a good indicator of sleepiness ranging from 1 to 9 where $1=$ very alert and $9=$ very sleepy, an effort to stay awake, fighting sleep (KSS) [12]. The sleep diary keeps track of the start and end time for each sleep period and subjective sleep quality rating on a 6-point Likert scale(where 1 =very good and 6=did not sleep).

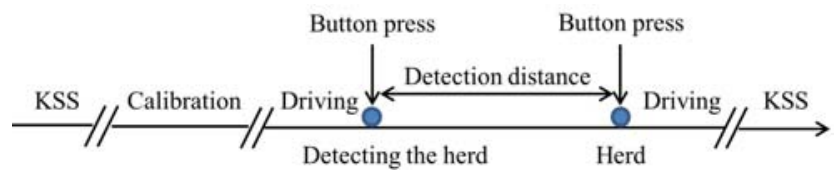

FIGURE I. EXPERIMENTAL TASK

\section{Procedure}

Two weeks before the experiment, participants were directed to operate the simulator under the guidance of a professional trainer. After two weeks' training, those participants who had a level of basic operation skill of train drivers could operate the train simulator skillfully.

Immediately after the training, participants took part in two driving tasks not more than 1 week apart, designed to produce varying levels of fatigue. In this study, sober driving was conducted at 9 am after the participant had a good sleep the night before. Fatigue driving was conducted at $14 \mathrm{pm}$ after the participant was deprived of 8 hours of sleep the night before. Both driving tasks were the same and lasted for 30 minutes. Half of the participants first completed the driving task in the morning, followed by driving task in the afternoon. The other half completed the two driving tasks in the opposite order. All participants were required to provide actual sleep condition of the night before the experiment in the sleep diary.

At the beginning and end of each driving, drivers were asked to complete subjective ratings of sleepiness using the KSS. After calibration of the eye tracker, drivers started the train and began the driving test. In the course of driving, the driver pressed the horn button as soon as he detected and passed the herd on the track. The experimental task is illustrated in Figure 1. The forward displacement of the train at the point where emergency horn commenced was recorded by the driving simulator. Vigilance performance and attention were measured by detection distance, which is defined as the track distance between detecting the herd and passing the herd. A longer detection distance indicated better vigilance and attention [9].

Eye movement data, including timestamp, gaze data, eye position, pupil size and data validity, was collected simultaneous by eye tracker during the driving task. A realtime eye movement data acquisition platform developed in C\# stored the eye data into database for following analysis.

\section{Data Processing}

Raw eye data was separated into segments using time window of 30 s and overlap is $50 \%$. When data loss occurs, -1 along with other physically impossible values is recorded by the eye tracker. Data segments containing more than $40 \%$ of missing data points or blinks with duration greater than $4 \mathrm{~s}$ within the analysis intervals were discarded [13].

\section{1) Pupil Diameter Preprocessing}

In a statistical perspective,-1 and other physically impossible values can be considered as outliers of the pupil diameter distribution. We replaced these outliers by means of linear interpolation. After that, a digital low-pass filter was applied to smooth the noise. Pupil data from both eyes were averaged.

\section{2) Blink Detection}

Blinks were detected as continuous sets of -1 output by the eye tracker. However, due to the participant's eyes' temporary reflection or the hardware systems transfer delays, the eye tracker also output -1 . In these cases the data loss is usually limited to a short period of time. To eliminate the effects of such values on blink detection, we discarded short blinks which were less than $75 \mathrm{~ms}$ [14]. Blinks separated by less than $100 \mathrm{~ms}$ were then merged as a single blink in line with Hupé et al [15].

\section{3) Fixation Extraction}

Our eye tracker uses Active Display Coordinate System (ADCS). X and Y coordinates are mapped into normalized unit ranging from 0 to 1 . In order to extract fixations from original gaze data, we applied Velocity Threshold Identification (I_VT) algorithm inspired by Komogortsev [14]. First, loss gaze data lower than $75 \mathrm{~ms}$ was filled by linear interpolation while longer than $75 \mathrm{~ms}$ (caused by blinks) was kept unprocessed. Second, a moving average filter was applied to smooth gaze data. Third, the velocity of gaze data was calculated by dividing the distance between two consecutive gaze data points by the time between two samples, as shown in

$$
v_{t_{1} t_{2}}=\frac{\left|S_{t_{1}}-s_{t_{2}}\right|}{\left|t_{1}-t_{2}\right|}
$$

Fourth, the velocity of gaze data was compared to the threshold. If the velocity was below than 1.8 unit/s, the corresponding gaze sample was marked as part of a fixation. Fifth, if the time between two consecutive fixations was below $75 \mathrm{~ms}$ and the distance was below 0.05 unit, they were merged into a longer fixation. Finally, fixations with duration less than $100 \mathrm{~ms}$ were discarded from the analysis.

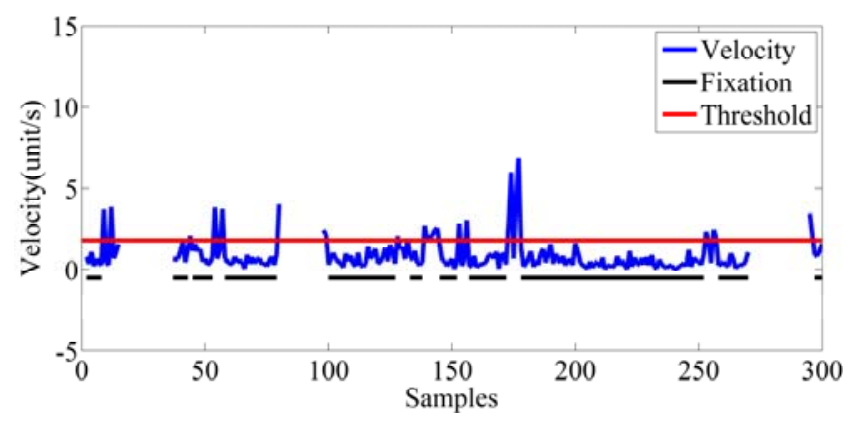

FIGURE II. THE RESULTS OF I-VT FIXATION CLASSIFICATION 
TABLE I. T-TEST RESULTS FOR SLEEP TIME, SLEEP QUALITY, KSS AND DETECTION DISTANCE BETWEEN TWO DRIVING TASKS

\begin{tabular}{ccccccc}
\hline \multicolumn{1}{c}{ Morning } & \multicolumn{2}{c}{ Afternoon } & \multicolumn{2}{c}{ t-test } \\
\hline & $\mathrm{M}$ & $\mathrm{SD}$ & $\mathrm{M}$ & $\mathrm{SD}$ & $\mathrm{t}$ & $\mathrm{p}$ \\
\hline Sleep time [h] & 9.01 & 0.63 & 1.50 & 0.55 & 33.54 & $\mathrm{P}<0.001$ \\
Sleep quality & 1.50 & 0.55 & 5 & 0.31 & -15.65 & $\mathrm{P}<0.001$ \\
$\begin{array}{c}\text { KSS } \\
\begin{array}{c}\text { Detection } \\
\text { distance [m] }\end{array}\end{array}$ & 2.67 & 0.82 & 7.17 & 0.98 & -7.99 & $\mathrm{P}<0.001$ \\
& 347.83 & 93.24 & 189.50 & 60.17 & 3.34 & $\mathrm{P}<0.001$
\end{tabular}

Using I_VT algorithm, we got fixations from original gaze data. An example of the result of fixation extraction algorithm is illustrated in Figure II. In the figure, the calculated velocity is indicated as a blue curve and the threshold is a red line. Blink onset and offset represent respectively the rapid increase and decrease of velocity. If the velocity is below than the threshold, the corresponding gaze sample is marked as part of single fixation. The detected fixations are marked by a broken line in black in the velocity chart.

\section{DATA ANALYSIS AND RESUlTS}

\section{A. Experimental Validation Results}

To compare the difference between two driving tasks (morning and afternoon), a t-test was performed to exam sleep time, sleep quality, KSS and detection distance. Data from all participants were combined together. The results of the t-test are shown in Table I.

The results show that sleep time, sleep quality, KSS and detection distance of drivers in the morning were significantly different from that in the afternoon $(p<0.001)$. Sleep deprivation induced fatigue, which further leads to larger KSS scores and decreased attention. As a result, the drivers' ability to detect abnormal events (such as herd on the track in our experiment) decreases, which means longer detection distance. That is to say, we can use subjective measures and detection distance as objective measures to indicate sleepiness. Thus, the morning and afternoon driving task can be considered as sober and fatigue driving respectively.

\section{B. Statistical Analysis of Eye movement Features}

For each participant, we extracted the mean pupil diameter, mean blink time and fixation percentage in 30s under sober and fatigue state as eye movement features. After feature extraction, we used a paired t-test to evaluate the difference between different driving state (sober, fatigue). Descriptive statistics of two driving states for each driver are shown in Table II.
The results show that the pupil diameter and fixation percentage of fatigue drivers was significantly different from that of sober drivers $(\mathrm{p}<0.001)$. Blink time was significantly different between the sober and fatigue driving state for most drivers $(p<0.001)$ except for subject \#2. These results prove that pupil diameter, blink time and fixation percentage are feasible features for high-way train drivers' fatigue detection and the reliability of the system can be improved with multiparameter fusion method. In fact, in tedious driving environment, drivers often relax themselves and may not pay much attention to the surroundings, which causes more blinks and less fixation percentages in fatigue.

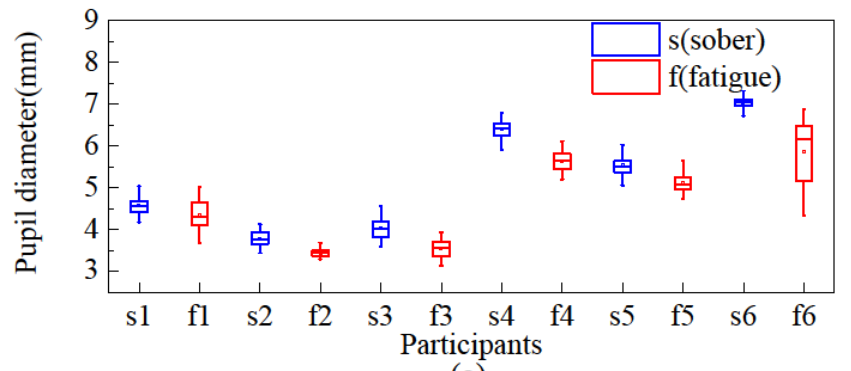

(a)

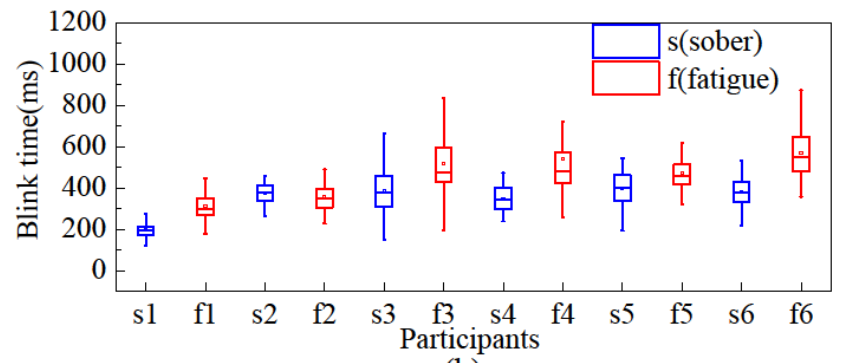

(b)

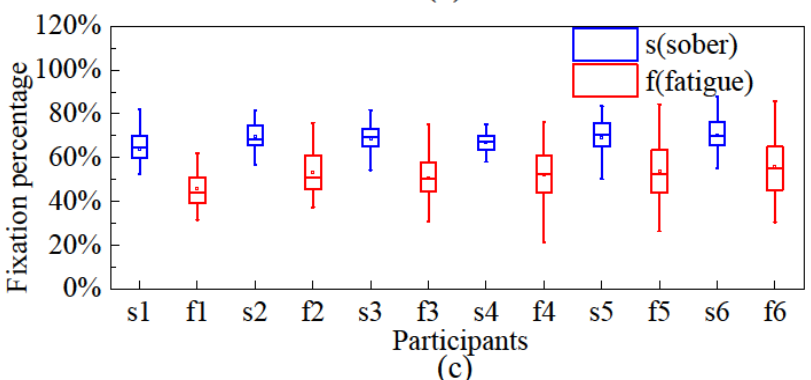

FIGURE III. FEATURE EXTRACTION FOR ALL PARTICIPANTS IN SOBER AND FATIGUE STATE

TABLE II. ESCRIPTIVE STATISTICS OF SOBER AND FATIGUE DRIVING STATES FOR EACH DRIVER

\begin{tabular}{|c|c|c|c|c|c|c|c|c|c|c|c|c|}
\hline \multirow[b]{2}{*}{ ubject } & \multicolumn{4}{|c|}{ Pupil diameter [mm] } & \multicolumn{4}{|c|}{ Blink time [ms] } & \multicolumn{4}{|c|}{ Fixation percentage [\%] } \\
\hline & $\begin{array}{c}\text { Sober } \\
(\mathrm{M} \pm \mathrm{SD})\end{array}$ & $\begin{array}{c}\text { Fatigue } \\
(\mathrm{M} \pm \mathrm{SD})\end{array}$ & $t$ & $p$ & $\begin{array}{l}\text { Sober } \\
(\mathrm{M} \pm \mathrm{SD})\end{array}$ & $\begin{array}{l}\text { Fatigue } \\
(\mathrm{M} \pm \mathrm{SD})\end{array}$ & $t$ & $p$ & $\begin{array}{c}\text { Sober } \\
(\mathrm{M} \pm \mathrm{SD})\end{array}$ & $\begin{array}{l}\text { Fatigue } \\
\qquad(\mathrm{M} \pm \mathrm{SD})\end{array}$ & $t$ & $p$ \\
\hline No.1 & $4.6 \pm 0.2$ & $4.4 \pm 0.4$ & 3.6 & $<0.001$ & $194.9 \pm 39.7$ & $317.2 \pm 65.0$ & -12.7 & $<0.001$ & $63.7 \pm 10.6$ & $46.8 \pm 10.9$ & 7.3 & $<0.001$ \\
\hline No.2 & $3.8 \pm 0.1$ & $3.4 \pm 0.1$ & 15.7 & $<0.001$ & $372.2 \pm 48.7$ & $354.8 \pm 61.5$ & 1.1 & 0.276 & $71.1 \pm 5.8$ & $53.1 \pm 9.5$ & 12.2 & $<0.001$ \\
\hline No.3 & $4.0 \pm 0.2$ & $3.5 \pm 0.2$ & 12.6 & $<0.001$ & $384.4 \pm 110.0$ & $517.6 \pm 159.4$ & -5.1 & $<0.001$ & $68.5 \pm 7.3$ & $50.7 \pm 11.4$ & 10.8 & $<0.001$ \\
\hline No.4 & $6.4 \pm 0.2$ & $5.6 \pm 0.2$ & 21.5 & $<0.001$ & $346.8 \pm 59.2$ & $541.5 \pm 217.6$ & -6.7 & $<0.001$ & $67.1 \pm 4.3$ & $52.5 \pm 12.1$ & 8.5 & $<0.001$ \\
\hline No.5 & $5.5 \pm 0.2$ & $5.1 \pm 0.2$ & 9.9 & $<0.001$ & $399.5 \pm 72.9$ & $474.5 \pm 81.9$ & -5 & $<0.001$ & $69.3 \pm 8.9$ & $54.0 \pm 15.0$ & 6.2 & $<0.001$ \\
\hline No.6 & $7.0 \pm 0.1$ & $5.9 \pm 0.8$ & 10.9 & $<0.001$ & $379.8 \pm 74.4$ & $568.8 \pm 181.9$ & -7.3 & $<0.001$ & $70.2 \pm 7.3$ & $56.1 \pm 13.8$ & 6.7 & $<0.001$ \\
\hline
\end{tabular}


Individual difference can be observed from Figure III. Figure III (a) gives the difference in pupil diameter between sober and fatigue during driving. The results show that the pupil diameter was lower and the distribution was broader in fatigue state compared to sober state for all participants. Variation range and pupil size were different across all participants in different driving states. Figure III (b) gives average blink time for each participant under sober and fatigue state. The results show that all participants except \#2 had longer average blink time in fatigue state. Different participants showed different individual characteristics under the same driving state. For example, larger mean and variation of blink time were observed from participant \#3 compared to participant $\# 1$.Figure III(c) compares the fixation percentage in sober and fatigue driving state. Fixation percentages of all participants were lower in fatigue state. Individual differences were mainly reflected in the variation of the fixation percentage. The individual difference may go beyond the difference caused by fatigue driving. Thus, personalized database for train drivers' fatigue identification should be established to ensure the reliability.

\section{CONCLUSION}

The study conducted a large amount of eye-gaze experiments in a bullet train driving simulator with subjects of different fatigue states. The presented results show that several analyzed eye movement features are good indicator for fatigue detection. Pupil diameter, blink time and fixation percentage of fatigue drivers are significantly different from that of sober drivers $(p<0.001)$. Thus, we can conclude that pupil diameter, blink time and fixation percentage can be used as metrics to measure train drivers' level of fatigue. Meanwhile, we reveal obvious individual difference of train drivers and propose personalized database hypothesis based on multi-parameter for train drivers' fatigue identification. These findings, as a preliminary study, are providing the theoretical basis for designing high-speed train driver fatigue monitor system.

\section{ACKNOWLEDGMENT}

This research was financially supported by Jiangsu Postgraduate Scientific Research and Innovation Projects under grant KYLX15_1239.

\section{REFERENCES}

[1] Härmä M, Sallinen M, Ranta R, et al. The effect of an irregular shift system on sleepiness at work in train drivers and railway traffic controllers[J]. Journal of sleep research, 2002, 11(2): 141-151.

[2] Australian Transport Safety Bureau (ATSB). Proceed authority exceeded by train 9104, Tarcoola, South Australia on 26 November 2012.

[3] Australian Transport Safety Bureau (ATSB). Derailment of Sydney Trains Passenger Train 602M near Edgecliff station, Sydney, NSW on 15 January 2014.

[4] Australian Transport Safety Bureau (ATSB). Wrong running direction involving passenger train 165-S, Mt Druitt, NSW on 12 March 2015.

[5] Gane N. Simulation[J]. Theory Culture \& Society, 2006, 23(2):250-252.

[6] Zoer I, Sluiter J K, Frings-Dresen M H. Psychological work characteristics, psychological workload and associated psychological and cognitive requirements of train drivers.[J]. Ergonomics, 2014, 57(10):1-15.

[7] Ingre M, Söderström M, Kecklund G, et al. Train drivers work situation. Working hours, sleep, stress and safety[J]. Stress Research Report, 2000, 292.
[8] Darwent D, Lamond N, Dawson D. The sleep and performance of train drivers during an extended freight-haul operation [J]. Applied Ergonomics, 2008, 39(5):614-22.

[9] Spring P, Mcintosh A, Caponecchia C, et al. Level of Automation: Effects on Train Driver Vigilance[J]. 2012.

[10] Phillips R O. What is fatigue and how does it affect the safety performance of human transport operators?[J]. 2014.

[11] Ji Q, Zhu Z, Lan P. Real-time nonintrusive monitoring and prediction of driver fatigue[J]. IEEE Transactions on Vehicular Technology, 2004, 53(4):1052-1068.

[12] Akerstedt T, Gillberg M. Subjective and objective sleepiness in the active individual.[J]. International Journal of Neuroscience, 1990, 52(12):29-37.

[13] Grandchamp R, Braboszcz C, Delorme A. Oculometric variations during mind wandering[J]. Frontiers in Psychology, 2014, 5(1):31-31.

[14] Komogortsev O V, Gobert D V, Jayarathna S, et al. Standardization of Automated Analyses of Oculomotor Fixation and Saccadic Behaviors[J]. IEEE Transactions on Biomedical Engineering, 2010, 57(11):2635 2645.

[15] Hupé J M, Lamirel C, Lorenceau J. Pupil dynamics during bistable motion perception.[J]. Journal of Vision, 2009, 9(7). 